\title{
Potential of Targeting Bone Metastases with Immunotherapies
}

\section{ISSN: 2637-773X}

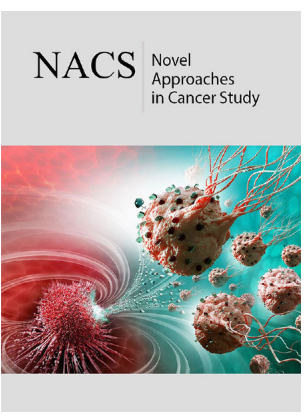

*Corresponding author: Kähkönen TE, Pharmatest Services Ltd, Itäinen Pitkäkatu 4C, 20400 Turku, Finland

Submission: 睯 September 18, 2019 Published: 制September 25, 2019

Volume 3 - Issue 3

How to cite this article: Kähkönen TE, Halleen JM, Bernoulli J. Potential of Targeting Bone Metastases with Immunotherapies. Nov Appro in Can Study. 3(3). NACS.000561.2019.

DOI: 10.31031/NACS.2019.03.000561

Copyright@ Kähkönen TE, This article is distributed under the terms of the Creative Commons Attribution 4.0 International License, which permits unrestricted use and redistribution provided that the original author and source are credited.

\author{
Kähkönen TE ${ }^{1 *}$, Halleen JM¹ and Bernoulli ${ }^{1}$ \\ ${ }^{1}$ Pharmatest Services Ltd, Finland
}

\begin{abstract}
Cancer-related bone metastases are incurable and cause high mortality in patients. Immunotherapies have been evaluated in large-scale clinical trials with advanced cancer patients, but effects on bone metastases have not been specified. This mini review introduces case reports where patients with bone metastases have been treated with immunotherapies and local effects on tumor growth have been assessed.
\end{abstract} Potential skeletal-related adverse effects of immunotherapies are also discussed.

Keywords: Metastatic cancer; Bone metastasis; Immunotherapies

\section{Introduction}

Cancer metastases cause high morbidity and mortality in patients. Bone metastases are most common in breast and prostate cancer, but they are also observed in many other cancers such as lung and renal cancer and melanoma [1]. In breast cancer the formation of metastases depends on the tumor subtype, and the major site for metastasis is the skeleton [2]. Patients with bone metastases have a 5-year survival rate of only $21 \%$ and a median survival time of 3 years. Prostate cancer is currently described as a bone disease due to high incidence of skeletal metastases. In prostate cancer patients with bone metastases, the 5-year survival rate is about $30 \%$ and the median survival time is 3 years [3]. Metastatic cancer patients are treated with conventional cancer therapies that are usually ineffective against bone metastases. Tumor-induced bone loss can also be treated with bone-targeting therapies. Bone marrow is an important immune organ that contains many immune cells, such as myeloid-derived suppressor cells, T cells, B cells and natural killer cells, and it is a cytokine rich microenvironment [4-6]. Immune cells can regulate many aspects of formation and growth of bone metastases [4]. Bone marrow is an immunosuppressive microenvironment, and immune suppressive cells in bone may promote tumor progression [5]. On the contrary, cytotoxic T cells and NK cells can be activated by immunomodulators to mediate anti-tumor effects. In addition, immune cells directly interact with bone cells, promoting tumor-induced effects on bone [6]

Immunotherapies, agents that activate patient's own dampened immune system to fight cancer, have also been applied to patients with metastases. Currently, programmed cell death 1 (PD-1), programmed death ligand 1 (PD-L1) and cytotoxic T-lymphocyte-associated protein 4 (CTLA-4) targeting therapies are approved for treatment of many advanced cancers. Information of immunotherapies' effects on bone metastases is still scarce but data is emerging from ongoing clinical investigations. It is important to understand that metastases differ from primary tumors, and there can be differences in metastases growing in different locations of the body. For example, a study of renal cell carcinoma indicated that expression of PD-1 and PD-L1 was increased in metastases compared to primary tumor [7]. Furthermore, a recent study showed discrepancy in the response to immunotherapy between metastases growing in different locations, with a poor overall response [8]. However, the expression of PD-L1 in different metastases, including bone metastases, was similar in non-small cell lung cancer [9].

Some reports show promising cases where individual patients with bone metastases have been cured with immunotherapies. One recent report showed that anti-PD-1 treatment decreased the growth of bone metastases in a patient with metastatic anorectal amelanotic melanoma [10]. Some bone metastatic prostate cancer patients treated with anti-CTLA-4, experienced a complete remission [11]. Similarly, a melanoma patient with bone metastases obtained a complete remission after treatment with PD-1 [12]. A prostate cancer patient with 
bone metastases undergoing alloreactive cytotoxic T lymphocyte therapy showed decreased serum prostate specific antigen levels, and the bone metastatic lesions diminished after two years of treatment [13]. Cancer-induced bone loss in patients with bone metastases is treated with bone-targeting therapies, typically with the receptor activator of nuclear factor kappa-B ligand (RANKL) antibody denosumab. Furthermore, targeting the RANKL-pathway can have immunological effects on tumors that may enhance the antitumor activity of immunotherapies [14]. Denosumab has been combined with immunotherapies in melanoma patients with bone metastases [15]. All of these patients responded to the treatments, showing promising efficacy.

Importantly, it should be recognized that immune activation may also have harmful effects on the skeleton. A recently published case series showed that immunotherapies were associated with skeletal related adverse effects including spinal cord compression, fractures and lesions caused by increased bone resorption [16]. Considering the biological relationship between immune and bone cells, it is likely that immunotherapies cause skeletal adverse effects, and bone safety evaluation should be considered especially before treating patients with bone metastases.

\section{Conclusion}

Even though there are promising case reports of patients with bone metastases successfully treated with immunotherapies, it is still controversial if these findings can be seen in large patient cohorts. There can be discordance between the expression of immune checkpoint markers and the response to immunotherapies in primary and metastatic tumors, which should be taken into consideration in patient selection and treatment planning. Also, possible skeletal-related adverse effects of immunotherapies should be carefully monitored and reported to recognize potential risk factors.

\section{Conflict of Interest}

Authors declare no conflicts of interest.

\section{References}

1. Sowder ME, Johnson RW (2019) Bone as a preferential site for metastasis. JBMR Plus 3(3): e10126.

2. Kast K, Link T, Friedrich K, Petzold A, Niedostatek A, et al. (2015) Impact of breast cancer subtypes and patterns of metastasis on outcome. Breast Cancer Res Treat 150(3): 621-629.
3. Logothetis C, Morris MJ, Den R, Coleman RE (2018) Current perspectives on bone metastases in castrate-resistant prostate cancer. Cancer Metastasis Rev 37(1): 189-196.

4. Janssen LME, Ramsay EE, Logsdon CD, Overwijk WW (2017) The immune system in cancer metastasis: Friend or foe? J Immunother Cancer 5(1): 79.

5. Reinstein ZZ, Pamarthy S, Sagar V, Costa R, Abdulkadir SA, et al. (2017) Overcoming immunosuppression in bone metastases. Crit Rev Oncol Hematol 117: 114-127.

6. Xiang L, Gilkes DM (2019) The contribution of the immune system in bone metastasis pathogenesis. Int J Mol Sci 20(4).

7. Zhang $X$, Yin $X$, Zhang H, Sun G, Yang $Y$, et al. (2019) Differential expressions of PD-1, PD-L1 and PD-L2 between primary and metastatic sites in renal cell carcinoma. BMC Cancer 19(1): 360.

8. Bilen MA, Shabto JM, Martini DJ, Liu Y, Lewis C, et al. (2019) Sites of metastasis and association with clinical outcome in advanced stage cancer patients treated with immunotherapy. BMC Cancer 19(1): 857.

9. Wang H, Agulnik J, Kasymjanova G, Fiset PO, Camilleri-Broet S, et al. (2019) The metastatic site does not influence PD-L1 expression in advanced non-small cell lung carcinoma. Lung Cancer 132: 36-38.

10. Tokuhara K, Nakatani K, Tanimura H, Yoshioka K, Kiyohara T, et al. (2017) A first reported case of metastatic anorectal amelanotic melanoma with a marked response to anti-PD-1 antibody nivolumab: A case report. Int J Surg Case Rep 31: 188-192.

11. Cabel L, Loir E, Gravis G, Lavaud P, Massard C, et al. (2017) Long-term complete remission with Ipilimumab in metastatic castrate-resistant prostate cancer: Case report of two patients. J Immunother Cancer 5: 31.

12. Kageyama SI, Yamaguchi S, Ito S, Suehara Y, Saito T, et al. (2016) A case report of using nivolumab for a malignant melanoma patient with rheumatoid arthritis. Int Cancer Conf J 5(4): 192-196.

13. Shi J, Chen Y, Chen Y, Shen Y, Zhao H, Sun H, et al. (2018) Alloreactive cytotoxic $\mathrm{T}$ lymphocyte immunotherapy treatment of a patient with metastatic prostate cancer: A case report. Medicine (Baltimore) 97(24): e11111.

14. Angela Y, Haferkamp S, Weishaupt C, Ugurel S, Becker JC, et al. (2019) Combination of denosumab and immune checkpoint inhibition: Experience in 29 patients with metastatic melanoma and bone metastases. Cancer Immunol Immunother 68(7): 1187-1194.

15. Peters S, Clezardin P, Marquez-Rodas I, Niepel D, Gedye C, et al. (2019) The Rank-rankl axis: An opportunity for drug repurposing in cancer? Clin Transl Oncol 21(8): 977-991.

16. Moseley KF, Naidoo J, Bingham CO, Carducci MA, Forde PM, et al. (2018) Immune-related adverse events with immune checkpoint inhibitors affecting the skeleton: A seminal case series. J Immunother Cancer 6(1): 104.

For possible submissions Click below:

Submit Article 